Textures and Microstructures, 1987, Vol. 7, pp. 115-129

Photocopying permitted by license only

(C) 1987 Gordon and Breach Science Publishers Inc.

Printed in the United Kingdom

\title{
Texture Analysis from Incomplete Pole Figures in Low Symmetry Cases
}

\author{
F. WAGNER and M. HUMBERT \\ Laboratoire de Métallurgie Structurale, Faculté des Sciences lle du Saulcy, \\ 57045 Metz Cedex, France
}

(Received 24 November 1986; in final form 12 December 1986)

From a classification of crystal symmetries involved in texture analysis we define a set of (low) crystal symmetries that can be similarly managed in the same computer program, using the harmonic method. A way of calculating the texture from incomplete pole figures is then proposed. Results are reported for two examples with hexagonal and trigonal crystal symmetry, respectively, and advantages and limitations of such texture analyses are discussed.

KEY WORDS: Texture analysis, crystal symmetries, harmonic method, incomplete pole figures.

\section{INTRODUCTION}

Various ways of calculating the O.D.F. from incomplete pole figures have been proposed for cubic materials. Conversely few results are reported for low symmetry materials, particularly for the harmonic method.

The aims of the present paper are first to survey the ways for such calculations, then to discuss their advantages and limitations and finally to present results obtained with a new program based on the most suitable concepts. Special attention will be paid to the possibility of considering a large range of crystal symmetries in the same formalism and consequently in the same program. 


\section{LOW SYMMETRIES}

The designation "low symmetry" is sometimes used in the field of texture analysis when the crystal symmetry considered is not that most-studied, i.e. cubic.

In order to define the symmetries which will be considered later let us recall the main points which concern texture analysis. Table 1 gives the list of the 32 crystal symmetry classes (or symmetry point groups); on each line are the classes which have the same rotational subgroup (given in the forth column). It is this rotational subgroup which has to be taken into account in texture calculations. The 32 classes are displayed in the first three columns which correspond respectively to one of these three cases:

i) the symmetry class does not include any inversion elements

ii) the symmetry class includes the center of symmetry $\overline{1}$ as element

iii) the symmetry class includes an inversion element but not $\overline{1}$ (m or $\overline{4}$ for example)

Table 1 Classification of the 32 symmetry point groups and their rotational subgroups

\begin{tabular}{|c|c|c|c|c|}
\hline \multicolumn{5}{|c|}{ The 32 symmetry point groups (SPG) } \\
\hline $\begin{array}{l}\text { The } 11 \mathrm{SPG}^{+} \\
\text {(enantio- } \\
\text { morphic) }\end{array}$ & $\begin{array}{l}\text { The } 11 \mathrm{SPG}^{-}(\overline{1}) \\
\text { (centro- } \\
\text { symmetric) }\end{array}$ & The $10 \mathrm{SPG}^{-}$ & $\begin{array}{l}\text { Rotational } \\
\text { subgroup }\end{array}$ & $\begin{array}{l}\text { Type of the } \\
\text { subgroup }\end{array}$ \\
\hline 1 & $\overline{1}$ & $\mathrm{~m}$ & 1 & $\mathrm{Cn}$ \\
\hline 2 & $2 / \mathrm{m}$ & $\overline{4} ; \mathrm{mm} 2$ & 2 & $\mathrm{Cn}$ or $\mathrm{Dn}^{\mathrm{a}}$ \\
\hline 3 & $\overline{3}$ & $\overline{6} ; 3 \mathrm{~m}$ & 3 & $\mathrm{Cn}$ \\
\hline 4 & $4 / \mathrm{m}$ & $4 \mathrm{~mm}$ & 4 & $\mathrm{Cn}$ \\
\hline 6 & $6 / \mathrm{m}$ & $6 \mathrm{~mm}$ & 6 & $\mathrm{Cn}$ \\
\hline $\begin{array}{r}222 \\
32 \\
422 \\
622\end{array}$ & $\begin{array}{l}\mathrm{mmm} \\
\overline{3} \mathrm{~m} \\
4 / \mathrm{m} \mathrm{mm} \\
6 / \mathrm{m} \mathrm{mm} \\
\end{array}$ & $\begin{array}{l}\overline{4} 2 \mathrm{~m} \\
\overline{6} \mathrm{~m} 2\end{array}$ & $\begin{array}{r}222 \\
32 \\
422 \\
622\end{array}$ & $\begin{array}{l}\text { Dn } \\
\text { Dn } \\
\text { Dn } \\
\text { Dn }\end{array}$ \\
\hline $\begin{array}{r}23 \\
432\end{array}$ & $\begin{array}{l}\mathrm{m} 3 \\
\mathrm{~m} 3 \mathrm{~m}\end{array}$ & $\overline{4} 3 \mathrm{~m}$ & $\begin{array}{r}23 \\
432\end{array}$ & $\begin{array}{l}\mathrm{T} \\
\mathrm{O}\end{array}$ \\
\hline
\end{tabular}

${ }^{a}$ Depends on the choice of setting the crystal coordinate system. 
The 11 classes of type i) concern enantiomorphic crystals. It was demonstrated that for such classes texture description requires two functions, one for right-handed crystals and one for left-handed crystals (Bunge, Esling and Muller, 1981; Esling, 1981). Because it is quite difficult in practice to obtain pole figures for the right- and left-handed forms separately, we will not consider later these symmetries although the mathematical formalism and the texture analysis program described here could be used. For symmetry classes of type ii) and iii) only one texture function is required (Bunge, Esling and Muller, 1981; Esling, 1981).

In the last column of Table 1 the type of the rotational subgroup is indicated (Cn denotes a cyclic group, Dn a dihedral one whereas $\mathrm{O}$ and $\mathrm{T}$ refer to octahedral and tetrahedral groups).

Assuming that the denomination low symmetry excludes the cases with octahedral and tetrahedral subgroups it can be seen from Table 1 that the remaining symmetry classes belong to either type $\mathrm{Cn}$ or Dn.

In the formalism of the harmonic method which requires the construction of symmetrized harmonic functions, denoted $k_{l}^{\mu}(\phi, \beta)$, it is easy to write formulae for $k_{l}^{\mu}(\phi, \beta)$ either in $\mathrm{Cn}$ or in Dn case. In $\mathrm{Cn}$ case a $\dot{k}_{l}^{\mu}(\phi, \beta)$ function is identical to a usual spherical harmonic function $k_{l}^{m}(\phi, \beta)$ :

$$
\dot{k}_{l}^{\prime \prime}(\phi, \beta)=k_{l}^{m^{\prime}}(\delta, \beta)
$$

with

$$
m^{\prime}=J m ; \quad-l \leqslant m \leqslant l
$$

Relation (2), where $J$ denotes the order of the highest order crystal axis taken as microscopic $Z$ axis, has been called a selection rule (Bunge, 1982). The index $\mu$ only denotes a new numbering of the functions and runs from 1 to $2 \cdot[l / J]+1$. In Dn case a $k_{l}^{\mu}(\phi, \beta)$ is defined by the sum of two harmonic functions:

$$
\dot{k}_{l}^{\mu}(\phi, \beta)=\varepsilon(\mu)\left(k_{l}^{m^{\prime}}(\phi, \beta)+(-1)^{l+m^{\prime}} k_{l}^{-m^{\prime}}(\phi, \beta)\right)
$$

with always the selection rule $m^{\prime}=J \cdot(\mu-1)$ and the normalization factor

$$
\varepsilon(\mu)=\left\{\begin{array}{lll}
\frac{1}{2} & \text { if } & \mu=1 \\
(2)^{-\frac{1}{2}} & \text { if } & \mu>1
\end{array}\right.
$$

Formula (3) is valid when the microscopic coordinate system ( $X Y Z)$ has its $Y$ axis along a twofold axis and $Z$ along a $J$-fold axis. 
One practical consequence is to simplify writing of a texture analysis program, in the harmonic method frame, for a set of symmetry classes of the same type. For example the program that we have used allows analysis of incomplete pole figures for materials with crystal symmetry of type Dn i.e. for the 6 classes standing in the framed part of Table 1.

\section{DETERMINATION OF THE NORMALIZATION FACTORS FOR POLE FIGURES}

In the case of incomplete pole figures the normalization factors, $\mathrm{Ni}$, are unknowns which have to be considered in addition to the usual unknowns of a texture analysis problem i.e. the coefficients of the series expansion of the O.D.F. in the frame of the harmonic method. The separation of the determination of the two kinds of unknowns into two stages of the calculation leads to a simplified and more efficient program.

Among the existing methods of evaluating the normalization factors (Kern and Bergmann, 1978; Humbert and Bergmann, 1980; Van Houtte, 1980; Dahms and Bunge, 1986) the two following are possible for low symmetries. The iterative method (Van Houtte, 1980) is not well adapted for these cases. It requires indeed a first approximation of each normalization factor based on the situation of a random distribution. This first approximation can be very bad in case of low-multiplicity pole figures. Moreover, because no mathematical criterion of convergency is known, it is difficult to decide how many steps must be carried out in the iterative procedure. The method proposed by Liang, Xu and Wang (1981) seems to us most suitable. We shall review it here. The formulae involved in the following hold for pole figures with triclinic specimen symmetry and small changes have been introduced with regard to the original work to take into account conventional methods of measurement.

Considering that pole figure values correspond to small cases along rings in the pole figure, the integrated pole density on a ring reads:

$$
{ }^{\mathrm{ex}} C_{h i}^{k}=N i \sum_{j} I_{h i}(j, k)\left(\cos \phi_{k}-\cos \phi_{k+1}\right) \Delta \beta
$$


where $h i$ denotes the considered pole figure, $j$ and $k$ are respectively the number of the case and the number of the ring, $I_{h i}(j, k)$ the measured intensity for this case, $\phi_{k}$ and $\phi_{k+1}$ the angular limits in tilt of the ring and $\Delta \beta$ the angular interval of the case in azimuth.

Equation (5) can be abbreviated with the following notation:

$$
{ }^{\mathrm{ex}} C_{h i}^{k}=N i^{\mathrm{ex}} \tilde{C}_{h i}^{k}
$$

where ${ }^{\text {ex }} \tilde{C}_{h i}^{k}$ is only related with known quantities.

Considering now a given O.D.F. which generates the pole density $P_{h i}(y)$ we define the integral th $C_{h i}^{k}$ on a given ring $k$ :

$$
{ }^{\text {th }} C_{h i}^{k}=\int_{\phi=\phi_{k}}^{\phi=\phi_{k+1}} \int_{\beta=0}^{\beta=2 \pi} P_{h i}(y) \sin \phi d \phi d \beta
$$

In the harmonic method the pole density $P_{h i}(y)$ can be expressed as a series depending on the coefficients $C_{1}^{\mu n}$ of the O.D.F.. This leads, for ${ }^{\text {th }} C_{h i}^{k}$, to the final expression:

$$
{ }^{\text {th }} C_{h i}^{k}=2 \pi\left(\cos \phi_{k}-\cos \phi_{k+1}+\sum_{l=2}^{l=l_{\max }}(8 \pi)^{\frac{1}{2}} G_{l}^{k} \sum_{\mu=1}^{\mu=M(l)} R C_{l}^{\mu 0} \hat{k}_{l}^{\mu}(h i)\right)
$$

where $R C_{l}^{\mu 0}$ are the real parts of the coefficients $C_{l}^{\mu 0}, \grave{k}_{l}^{\mu}(h i)$ the values of the symmetrized functions for $h i$, and $G_{l}^{k}$ are mathematical quantities:

$$
G_{l}^{k}=\int_{\phi=\phi_{k}}^{\phi=\phi_{k+1}} \bar{P}_{l}^{0}(\phi) \sin \phi d \phi
$$

with $\bar{P}_{l}^{0}(\phi)$ the associated Legendre polynomial as defined by Bunge (1982).

The normalization factors $\mathrm{Ni}$ are then obtained by a least squares method starting from the minimization condition:

$$
\sum_{i=1}^{i=1} \sum_{k=1}^{k=K}\left({ }^{\mathrm{th}} C_{h i}^{k}-N i^{\mathrm{ex}} \tilde{C}_{h i}^{k}\right)^{2}=\operatorname{Min}
$$

$I$ is the number of measured pole figures and $K$ the number of rings (assumed to be the same in all pole figures).

As is usual in the least squares method partial derivatives with respect to each unknown are set to zero. In our case this leads to a linear system of equations, with the $R C_{l}^{\mu 0}$ and $N i$ as unknowns, which can be solved by standard inversion programs. 
The number of unknowns, $U$, is indeed small and is given by:

$$
U=I+\sum_{l=2}^{l=l \max } M(l)
$$

where $M(l)$ is the number of symmetrized functions at a given rank $l$. For example $U$ is equal to 20 for an analysis with 3 pole figures and $l \max =16$ for an hexagonal material.

An important point about this method of determining the normalization factors $N i$ is that the minimization condition (10) is applied to the quantities $C_{h i}^{k}$ which are already sums of measurements on a ring. For this reason the influence of statistical errors on the measurements is considerably reduced.

Results for $\mathrm{Ni}$ factors which will be presented in section 5 are calculated by this method.

\section{DETERMINATION OF THE COEFFICIENTS $c_{l}^{\mu n}$ OF THE O.D.F.}

Assuming that normalization factors are determined we now have to minimize the difference of the following quantities:

- the "experimental" pole densities $P_{h i}^{\text {ex }}(y)$ which read:

$$
P_{h i}^{\mathrm{ex}}(y)=N i I_{h i}(y)
$$

with $I_{h i}(y)$ the intensity measured for the case centered on $y=\{\phi, \beta\}$.

- the "theoretical" pole densities $P_{h i}^{\text {th }}(y)$ which are related to the coefficients $C_{l}^{\mu n}$ of the O.D.F. through:

$$
\begin{aligned}
P_{h i}^{\mathrm{th}}(y)= & 1+\sum_{\substack{l=2 \\
(2) \\
l=l_{\max }}}^{n=l}(4 \pi /(2 l+1)) \varepsilon(n) \bar{P}_{l}^{n}(\phi) \\
& \times \sum_{\mu=1}^{\mu=M(l)} \dot{k}_{l}^{\mu}(h i)\left(R C_{l}^{\mu n} \cos n \beta-I C_{l}^{\mu n} \sin n \beta\right)
\end{aligned}
$$

where $R C_{l}^{\mu n}$ and $I C_{l}^{\mu n}$ denote the real and imaginary part of $C_{l}^{\mu n}$, $\bar{P}_{l}^{n}(\phi)$ an associated Legendre polynomial as defined by Bunge 
(1982) and:

$$
\varepsilon(n)=\left\{\begin{array}{lll}
(2 \pi)^{-1 / 2} & \text { if } & n=0 \\
(-1)^{n}(2 / \pi)^{1 / 2} & \text { if } & n \neq 0
\end{array}\right.
$$

Details about this formalism can be found in the work of Wagner (1983) and in Quantitative Texture Analysis (1982).

The determination of the unknown $C_{l}^{\mu n}$ is obtained in the same way by solving systems of linear equations resulting from a minimization condition.

This condition reads:

$$
\sum_{i=1}^{i=I} \int_{\phi=0}^{\phi=\phi_{\max }} \int_{\beta=0}^{\beta=2 \pi}\left(P_{h i}^{\mathrm{th}}(y)-P_{h i}^{\mathrm{ex}}(y)\right)^{2} d y=\operatorname{Min}
$$

where $\phi_{\max }$ denotes the maximum tilt angle (assumed to be the same for each pole figure).

As already noted by Morris (1975) and Humbert and Bergmann (1980) the set of linear equations derived from (15) can be split into several subsets of equations because of orthogonality properties of the exponential term of associated Legendre polynomials $\bar{P}_{l}^{n}(\phi)$ when $\beta$ runs from 0 to $2 \pi$. Each subset contains, as unknowns, the coefficients $C_{l}^{\mu n}$ with a fixed $n$ value. There are $(l \max +1)$ subsets with a decreasing number of unknowns when $n$ varies from 0 to $l$ max. When considering the programming it is an advantage of the harmonic formalism which leads to several subsets of equations rather than to one large system containing all the unknowns as is the case in the vector method.

For example a very sharp texture in hexagonal materials which would require an order $l \max =28$ leads to a matrix of rank 39 in the largest subset.

This formalism leads to the determination of the coefficients $C_{l}^{\mu n}$ with even $l$ i.e. to the even part $\tilde{f}(g)$ of the O.D.F. The search of the odd part $\tilde{\tilde{f}}(g)$, the so-called "ghost correction", can then be performed as usual by using the zero range method (Bunge and Esling, 1979; Bechler-Ferry, Bunge and Esling, 1981) or a Gauss fit (Lücke et al., 1981) for example.

\section{EXAMPLES OF ANALYSIS AND DISCUSSION}

A new program of texture analysis was developed according to the previously explained concepts. In order to discuss the accuracy and 

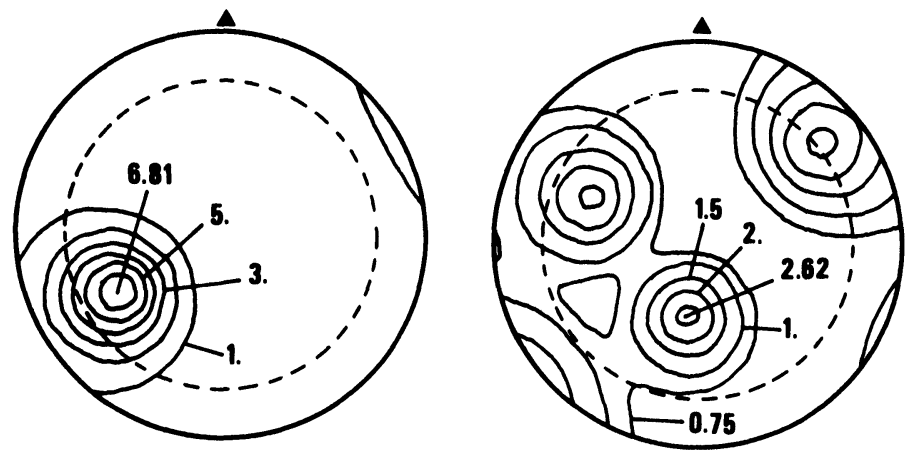

(00.6)

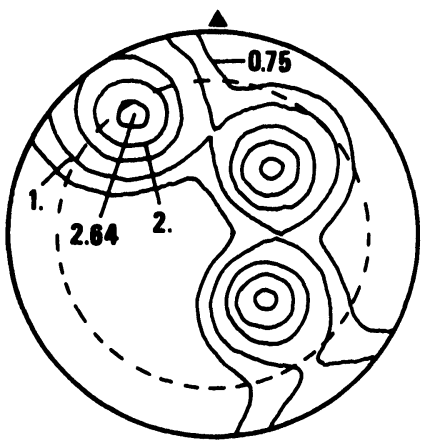

(10.4)

(20.2)

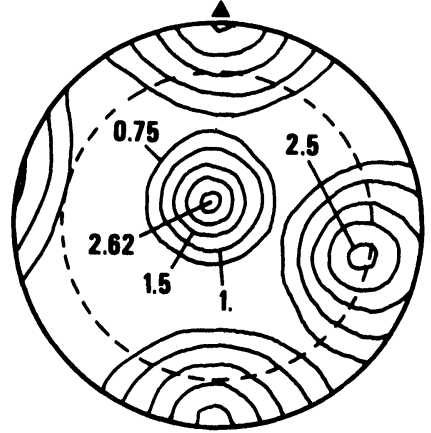

(01.2)
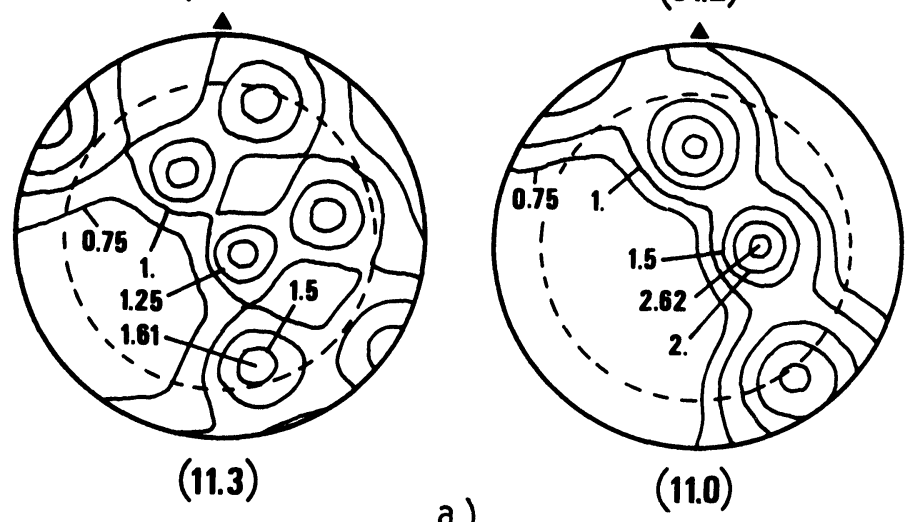

a)

Figure 1 Texture $T$ (trigonal-triclinic symmetries). (a) The 6 "experimental" pole figures $\left(\phi_{\max }=75^{\circ}\right.$ is indicated with the dashed circle). (b) The same pole figures recalculated after the analysis. 

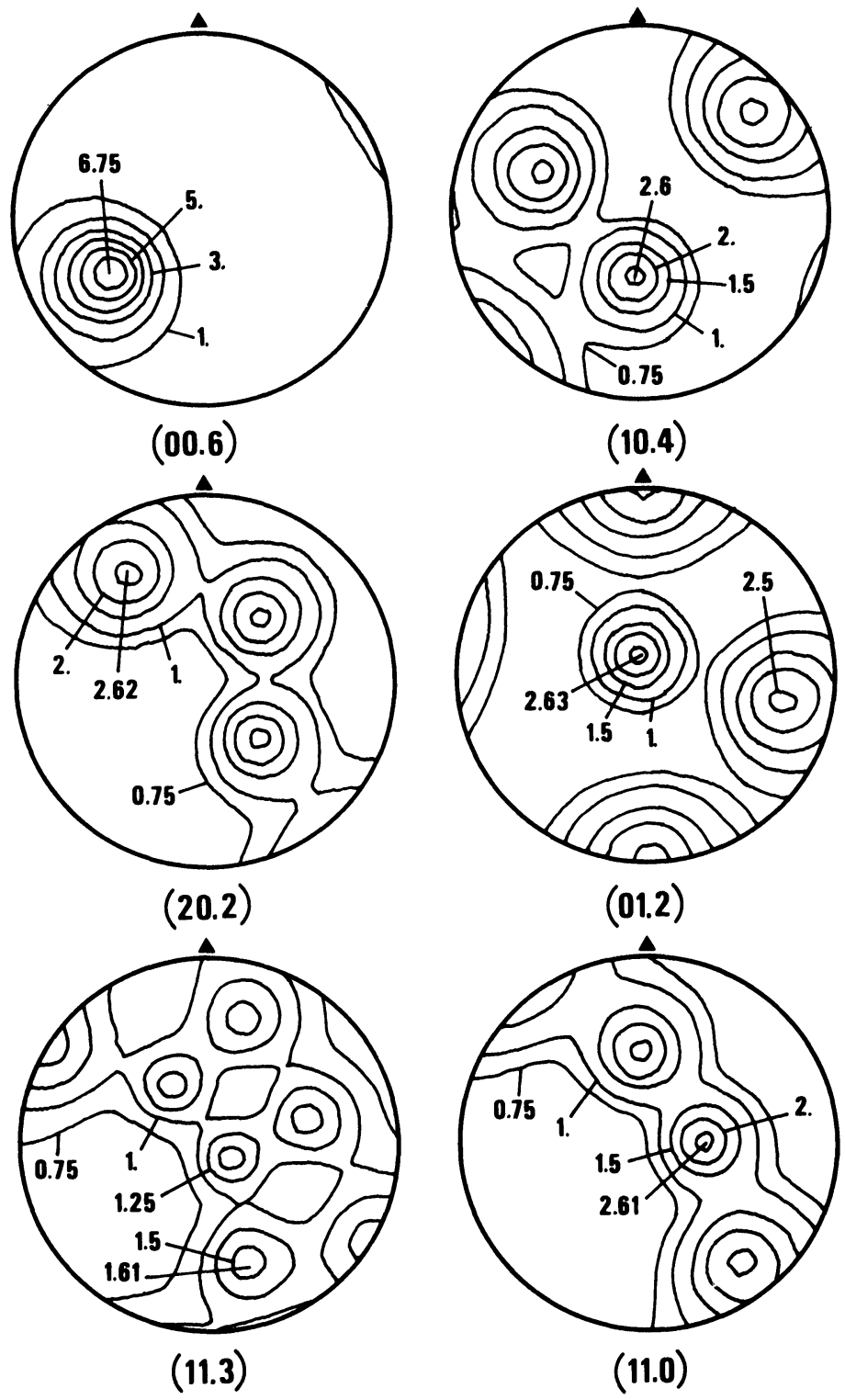

b)

Figure 1 (contd.) 
the limitations of such analysis two examples are presented, the first with simulated data the second with real measurements. In the first case the O.D.F. with trigonal-triclinic symmetries is known and consists of a Gaussian peak and a constant part (it will be denoted texture $T$ in the following); 6 pole figures were calculated using a program written by Matthies, Vinel and Helming (1980) and could

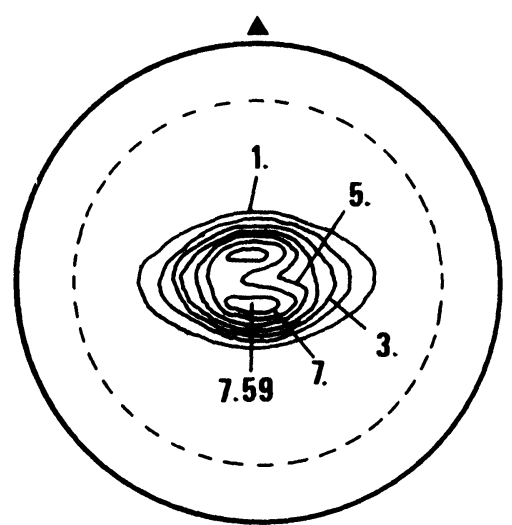

(00.2)

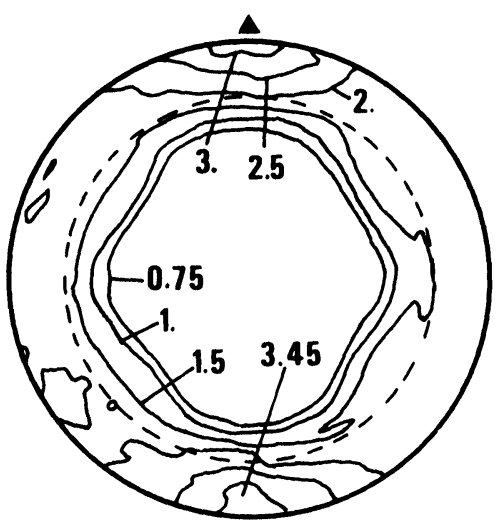

(10.0)

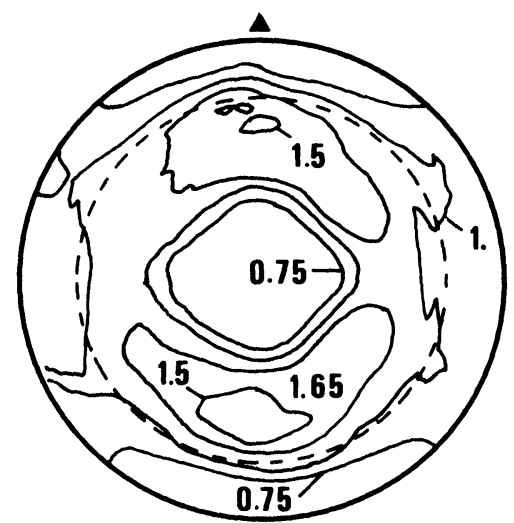

(10.1)

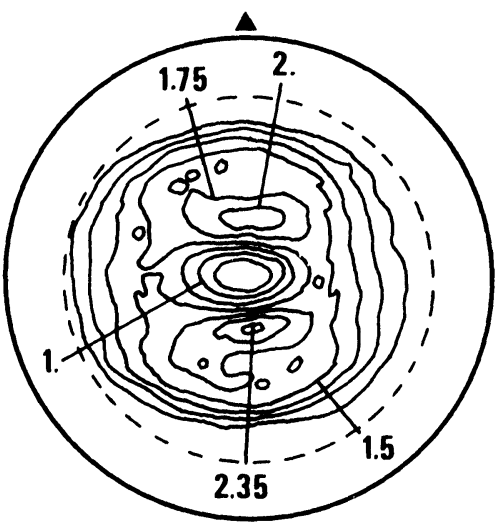

(10.2)

a)

Figure 2 Texture $R$ (hexagonal-triclinic symmetries). (a) The 4 experimental pole figures $\left(\phi_{\max }=75^{\circ}\right.$ is indicated with the dashed circle). (b) The same pole figures recalculated after the analysis. 
then be used as input data (Figure 1a). In the second case, denoted in the following as texture $R$, the data consist in four complete pole figures (Figure 2a), measured with back reflexion and transmission techniques, corresponding to a cold rolled Zircaloy 4 sheet (hexagonal-triclinic symmetries are considered).

Complete pole figures are then known in both cases but only rings up to a given tilt angle $\phi_{\max }$ are used in the texture analyses. Figure

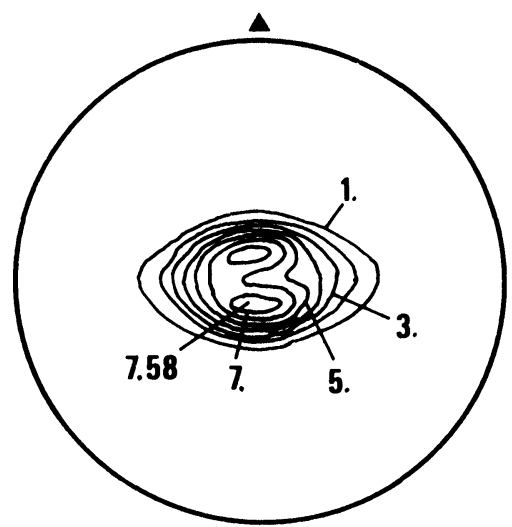

(00.2)

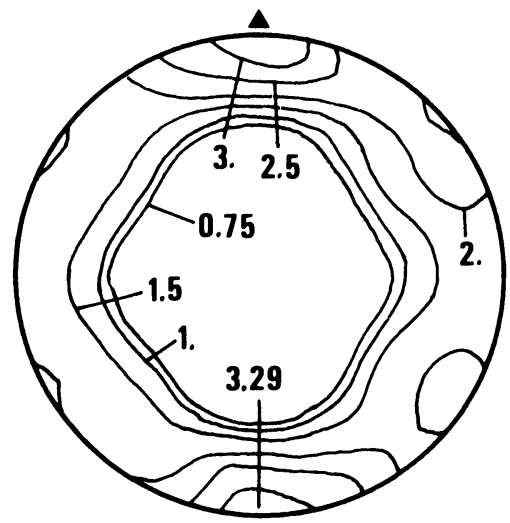

(10.0)

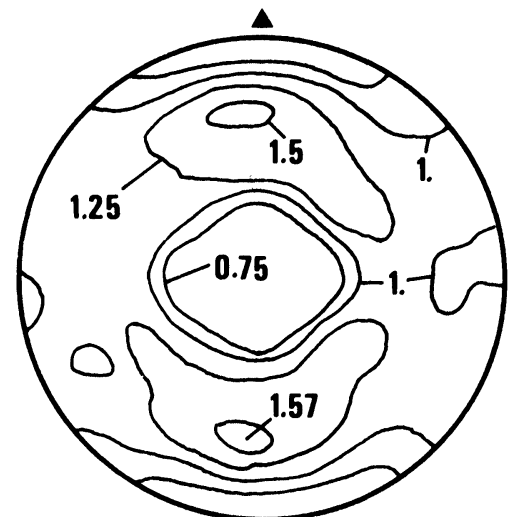

(10.1)

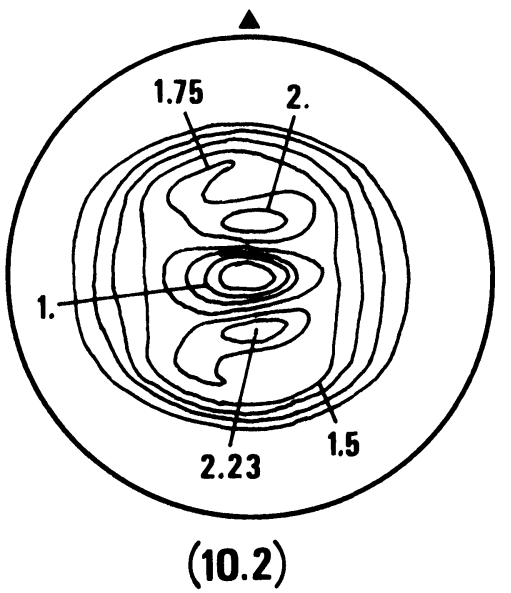

b) 
$1 b$ and $2 b$ show the pole figures recalculated from the O.D.F. coefficients in order to permit visual comparison. In both cases the analysis is performed up to $l \max =16$ and for $\phi_{\max }=75^{\circ}$ which is symbolized in Figures $1 \mathrm{a}$ and $2 \mathrm{a}$ with a dashed circle. Such analyses have been worked out for various $\phi_{\max }$ and results are discussed taking into account the normalization factors and error coefficients.

Figure $3 \mathrm{a}$ and $3 \mathrm{~b}$ show the evolution of the mean relative "error" of the normalization factors $\overline{\Delta N i} / N i$ defined as:

$$
\frac{\overline{\Delta N i}}{N i}=(1 / I) \sum_{i=1}^{i=I}\left(N i\left(\phi_{\max }=90^{\circ}\right)-N i\left(\phi_{\max }\right)\right) / N i\left(\phi_{\max }=90^{\circ}\right)
$$

It should be noted that the term error is only valid for the texture $T$ where the quantities $N i\left(\phi_{\max }=90^{\circ}\right)$ are known exactly, whereas in texture $R$ these factors $N i\left(\phi_{\max }=90^{\circ}\right)$ are calculated from the measured complete pole figures and are then only an approximation of the unknown true values. Figure $3 a$ and $3 b$ are then to be considered mainly as a help to discuss the stability of the calculation of normalization factors.

Although at different levels, the variation of the mean error $\overline{\Delta N i} / N i$ with decreasing $\phi_{\max }$ (i.e. with an increasing loss of information) is very low; for example $\overline{\Delta N i} / N i$ is less than $0.05 \%$ for texture $T$ and less than $5 \%$ for texture $R$ when $\phi_{\max }=65^{\circ}$.

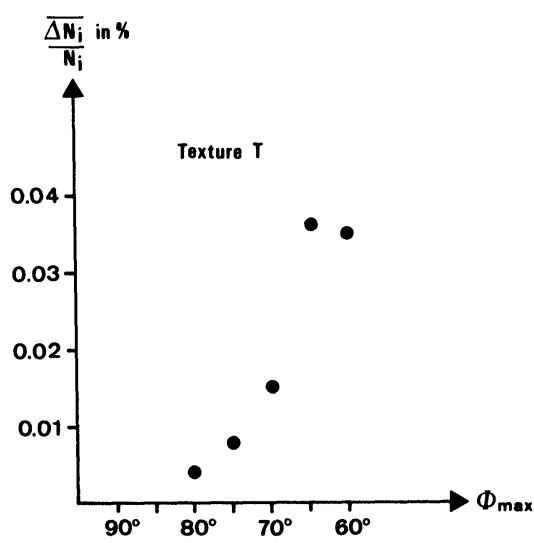

a)

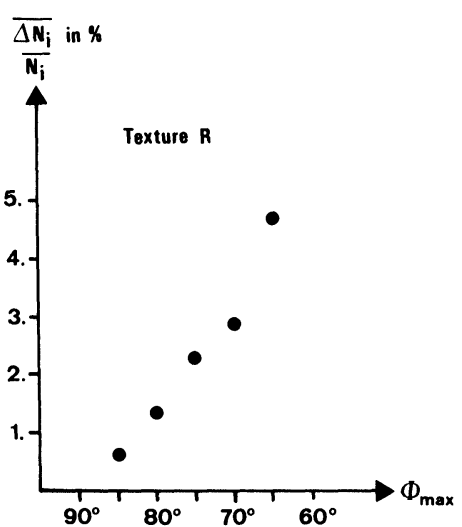

b)

Figure 3 The mean relative error for normalization factor $\overline{\Delta N i} / N i$ versus the maximal tilt angle $\phi_{\max }$. (a) Texture T. (b) Texture $R$. 
Our conclusion on this point is that the calculation of the normalization factors is not very sensitive to the maximum tilt angle $\phi_{\max }$, at least for pole figures without very sharp peaks, which is usually the case for low-symmetry materials.

The quality of a texture analysis can be characterized by error coefficients obtained from the comparison of experimental and recalculated pole-figure values. Hereafter the following are used:

$$
\operatorname{ERN} 1(h i)=\left(100 / N_{t}\right) \sum_{k}\left|P_{h i}^{\mathrm{ex}}\left(y_{k}\right)-P_{h i}^{\mathrm{rec}}\left(y_{k}\right)\right|
$$

where $y_{k}$ defines the center of a case in a pole figure and $N_{t}$ the total number of the cases in a pole figure.

$$
\mathrm{RP}(h i)=\left(100 / N_{t}\right) \sum_{k}\left|P_{h i}^{\mathrm{ex}}\left(y_{k}\right)-P_{h i}^{\mathrm{rec}}\left(y_{k}\right)\right| / P_{h i}^{\mathrm{ex}}\left(y_{k}\right)
$$

(if $p_{h i}^{\text {ex }}\left(y_{k}\right)$ is less than 0.01 the corresponding term is not taken into account and $N_{t}$ is decreased by one unit).

It should be noted that for the present examples $N_{t}$ is always the total number of cases of the complete pole figures which are known from calculation (texture $T$ ) or measurements (texture $R$ ). They are respectively the mean absolute "error" (multiplied by 100) and the mean relative "error" for a given $h i$ pole figure. ERN1 and $\overline{R P}$ denote their mean values for the whole set of pole figures which are used in a given texture analysis. Figure $4 \mathrm{a}$ and $4 \mathrm{~b}$ summarize the values of $\overline{E R N 1}$ and $\overline{R P}$ for several analyses as a function of $\phi_{\max }$. For texture $T$ these error coefficients increase with decreasing $\phi_{\max }$ with a sudden increase below $\phi_{\max }=65^{\circ}$. This increase is easily understood by considering the pole-figures: when $\phi_{\max }$ is less than $65^{\circ}$ only one peak among the three symmetrically equivalent ones is taken into account in pole figure (01.2); this pole figure no longer furnishes useful information. The situation is almost the same for pole figure (00.6) where the main part of the peak becomes located in the unused part of the pole figure when $\phi_{\max }=60^{\circ}$.

In the case of texture $R$ the results for the coefficients $\overline{\text { ERN1 }}$ and $\overline{\mathrm{RP}}$ (Figure 4b) are more unexpected. After a low increase with decreasing $\phi_{\max }$ they fall to minimal values at $\phi_{\max }=70^{\circ}$ before increasing very fast. This indicates that the analysis with incomplete pole figures (for $\phi_{\max }=70^{\circ}$ ) is better than that which uses complete pole figures. It is not really a surprise when one remembers that complete pole figures require the adjustment of reflexion and 


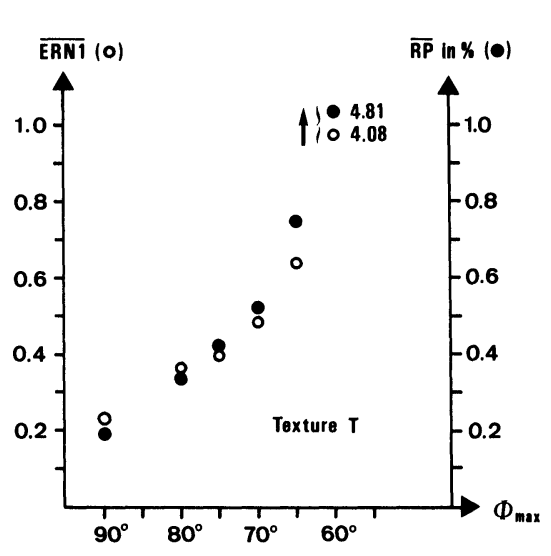

a)

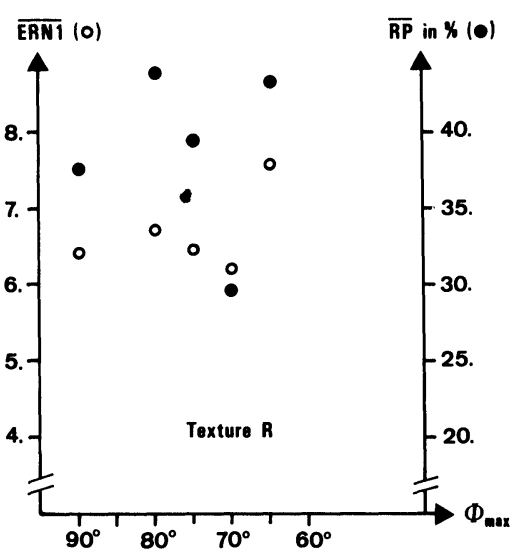

b)

Figure 4 The mean error coefficients $\overline{\mathrm{ERN} 1}$ and $\overline{\mathrm{RP}}$ versus the maximal tilt angle $\phi_{\max }$ (a) Texture T. (b) Texture $R$.

transmission X-ray measurements together with several kinds of corrections (background, defocussing, variation of diffracting volume and absorption) which can lead to falsification of the data (Humbert, 1986).

As it was already emphasized for cubic materials there is a "limit" for $\phi_{\max }$, i.e. in the loss of information, beyond which the errors increase drastically (Pospiech and Jura, 1974; Van Houtte, 1980). Obviously this "limit" is linked with the texture itself as illustrated previously with texture $T$. By considering the multiplicity of pole figures which are usually available in low symmetry materials and the examples presented, this "limit" can be located within the range $65-75^{\circ}$ for $\phi_{\max }$.

\section{CONCLUSION}

We hope to succeed in demonstrating that for incomplete pole figures:

- the harmonic method permits formulation of the texture analysis problem for a wide range of symmetries (for the so-called 
"low symmetries") and writing one texture analysis program for such cases.

- the values of the normalization factors calculated from a criterion of the "Liang et al. type" are nearly correct and vary slowly with the maximal tilt angle $\phi_{\max }$.

- the analyses are satisfactory as long as $\phi_{\max }$ is greater than a "limit" value located within the range $65-75^{\circ}$.

\section{References}

Bechler-Ferry, E., Bunge, H. J. and Esling, C. (1981). Proceedings of ICOTOM 6, the iron and steel Institute of Japan ed., II, p. 1359.

Bunge, H. J., (1982). "Texture Analysis in Materials Science", ButterworthsLondon ed.

Bunge, H. J. and Esling, C. (1979). J. Phys. Lett., 40, p. L-627.

Bunge, H. J., Esling, C. and Muller, J. (1981). Acta Cryst., A37, p. 889.

Dahms, M. and Bunge, H. J. (1986). Textures and Microstructures, 6, p. 167.

Esling, C. (1981). Thèse de doctorat d'état, University of Metz (France).

Humbert, M. (1986). In "Experimental Techniques of Texture Analysis", Bunge, H. J. ed., DGM Verlag, p. 29.

Humbert, M. and Bergmann, H.W. (1980). J. Appl. Cryst., 13, p. 500.

Kern, R. and Bergmann, H. W. (1978). Proceedings of ICOTOM 5, Gottstein G. and Lücke, K. ed, Springer Verlag, I, p. 139.

Liang, Z. Xu, J. and Wang, F. (1981). Proceedings of ICOTOM 6, the Iron and steel Institute of Japan ed., II, p. 1259.

Lucke, K., Pospiech, J., Virnich, K. H. and Jura, J. (1981). Acta Met., 29, p. 167.

Matthies, S., Vinel, G. and Helming, K. (1980). "Standard distributions in texture analysis", Akad. Verlag-Berlin.

Morris, P. R. (1975). Adv. X-Ray Anal., 18, p. 514.

Pospiech, J. and Jura, J. (1974). Zeit. Metallkd., 65, p. 324.

Quantitative Texture Analysis, Bunge, H. J. and Esling, C. (1982). ed, D. G. M. Oberusel.

Van Houtte, P. (1980). Mater. sci. eng., 43, p. 7.

Wagner, F. (1983). Thèse de doctorat d'état, University of Metz (France). 